\title{
VIOLÊNCIA SEXUAL CONTRA MULHERES PORTADORAS DE NECESSIDADES ESPECIAIS: PERFIL DA VÍTIMA E DO AGRESSOR
}

Rafaella Queiroga Soutoํㅡ, Carla Carolina da Silva Leite², Inácia Sátiro Xavier de França³, Alessandro Leite Cavalcanti ${ }^{4}$

RESUMO: Estudo transversal e descritivo caracterizou 19 vítimas de violência sexual portadores de necessidades especiais e agressores entre 396 casos registrados no período de 2004 e 2009 em Campina Grande - Paraíba. Os dados foram coletados por pesquisadores treinados, registrados em instrumento estruturado elaborado para o estudo e testado previamente; organizados e analisados com auxílio do software Statistical Package for the Social Sciences 17.0 e estatística descritiva. Verificou-se que 4,8\% dos casos registrados envolveram mulheres portadoras de necessidades especiais. As vítimas, na maioria com deficiência intelectual, eram solteiras, com idade entre 12 e 19 anos e baixa escolaridade. O agressor era em sua maioria único e conhecido da vítima; constatou-se o emprego de constrangimento em $21,1 \%$ dos casos, sendo a força física e a grave ameaça as mais frequentes. As equipes de saúde podem contribuir para a prevenção e detecção da violência sexual, com atenção a portadores de necessidades especiais. PALAVRAS-CHAVE: Pessoas com deficiência; Violência contra a mulher; Violência sexual; Estupro; Enfermagem.

\section{SEXUAL VIOLENCE AGAINST WOMEN WITH SPECIAL NEEDS: PROFILES OF VICTIMS AND AGGRESSORS}

\begin{abstract}
This transversal and descriptive study describes 19 special needs victims of sexual violence and aggressors from 396 cases of sexual violence registered in the period 2004 to 2009 in Campina Grande in the state of Paraíba. The data was collected by trained researchers and recorded on a structured research instrument developed for the study and previously tested. Data was organized and analyzed with the help of the software Statistical package for the Social Sciences 17.0 and descriptive statistics. It was ascertained that $4.8 \%$ of the cases of sexual violence involved women with special needs. The victims, the majority of whom had mental disabilities, were single, aged between 12 and 19, and with little schooling. The majority of the aggressors were known to the victim and acted alone; it was established that violence was used in $21.1 \%$ of cases, with physical violence and serious threats being most common. Health teams can contribute to the prevention and detection of sexual violence, with particular regard to people with special needs.
\end{abstract}

KEYWORDS: Handicapped; Violence against women; Sexual violence; Rape; Nursing.

\section{VIOLENCIA SEXUAL CONTRA MUJERES PORTADORAS DE NECESIDADES ESPECIALES: PERFIL DE LA VÍCTIMA Y DEL AGRESOR}

RESUMEN: Estudio transversal y descriptivo que caracterizó 19 víctimas de violencia sexual portadores de necesidades especiales y agresores entre 396 casos registrados en el periodo de 2004 y 2009 en Campina Grande - Paraíba. Los datos fueron recogidos por investigadores profesionales, registrados en instrumento estructurado elaborado para el estudio y evaluados previamente; organizados y analizados con el auxilio del software Statistical Package for the Social Sciences 17.0 y estadística descriptiva. Se verificó que $4,8 \%$ de los casos involucran mujeres portadoras de necesidades especiales. Las víctimas, en la mayoría con deficiencia intelectual, eran solteras, con edad entre 12 y 19 años y poca escolaridad. El agresor era en su mayoría único y conocido de la víctima; se ha constatado el empleo de violencia en $21,1 \%$ de los casos, siendo la fuerza física y la grave amenaza las más frecuentes. Los equipos de salud pueden contribuir para la prevención y detección de la violencia sexual, con atención a portadores de necesidades especiales.

PALABRAS-CLAVE: Personas con deficiencia; Violencia contra la mujer; Violencia sexual; Estupro; Enfermería.

\footnotetext{
${ }^{1}$ Enfermeira. Doutoranda em Enfermagem pelo Programa de Pós-Graduação em Enfermagem da Escola de Enfermagem da Universidade de São Paulo.

${ }^{2}$ Acadêmica do Curso de Graduação em Enfermagem da Universidade Estadual da Paraíba - UEPB.

${ }^{3}$ Enfermeira. Doutora em Enfermagem. Professora Departamento de Enfermagem e Programa de Pós-Graduação da UEPB. Bolsista de Produtividade do CNPq.

${ }^{4}$ Odontólogo. Doutor em Odontologia. Professor do Departamento de Odontologia da UEPB.
}

Autor correspondente:

Recebido: 29/06/2011

Rafaella Queiroga Souto Aprovado: 27/01/2012

Universidade de São Paulo

Av. Dr. Enéas de Carvalho Aguiar, 419 - 05403-000 - São Paulo-SP-Brasil

E-mail: soutorafaellaqueiroga@usp.br 


\section{INTRODUÇÃO}

A violência contra mulheres é um crime frequente e consiste em todo ato agressivo baseado na diferença de gênero, que resulte em sofrimentos e danos físicos, sexuais e psicológicos para a mulher; inclusive ameaças de tais atos, coerção e privação da liberdade, seja na vida pública ou privada. Este tipo de violência pode ser praticada por meio de abuso, estupro ou atentado violento ao pudor e atinge mulheres de qualquer faixa etária, classe social e etnia; muitos agressores são conhecidos das vítimas e, em alguns casos, fazem parte do ciclo familiar ${ }^{(1)}$.

No Brasil, as altas taxas de violência contra a mulher e a necessidade de legislação específica resultaram na criação da lei federal n. 10.778/2003 que estabelece a notificação compulsória dos casos, seja a mulher atendida em serviços públicos ou privados. Três anos depois, foi promulgada a Lei n. 11.340/2006, conhecida como Lei Maria da Penha, que estabelece mecanismos para coibir a violência doméstica e familiar contra a mulher, nos termos do $\S 8^{\circ}$ do art. 226 da Constituição Federal ${ }^{(2)}$.

Nessa luta antiviolência o respeito aos Direitos Humanos requer a promoção da vida em sociedade, sem discriminação de classe social, de cultura, de religião, de raça, de etnia ou de orientação sexual. E para que exista a igualdade de direitos, é preciso respeito às diferenças. Essa ideia contribui com a compreensão de que as pessoas com deficiência têm, naturalmente, o direto de desenvolver sua sexualidade e de serem respeitadas ${ }^{(3)}$.

As pessoas em situação de paraplegia ou tetraplegia ou, ainda, amputação de membro, têm inabilidade para a defesa pessoal devido à falta de locomoção. Aquelas com deficiência intelectual, também, devido ao comprometimento da área linguístico-cognitiva, podendo ser facilmente seduzidas tendo em vista que quanto maior o vazio no domínio de linguagem, menor é a consciência acerca da realidade do mundo, da noção do certo e errado, e principalmente, do despertar de sua sexualidade ${ }^{(4)}$.

Em se tratando das pessoas com deficiência auditiva, estas apresentam problemas perceptíveis na fala e se mostram indefesas a ataques sexuais, uma vez que não conseguem comunicação satisfatória, tanto para sua defesa frente ao ataque, como para denunciar os fatos às autoridades. Esses indivíduos se tornam susceptíveis aos agressores, que contam com a omissão da denúncia e a impunidade ${ }^{(5)}$.

Por outro lado, as pessoas com deficiência visual nem sempre são compreendidas, pois utilizam a linguagem gestual para se comunicar, que não faz parte do cotidiano da população em geral ${ }^{(6)}$, além disso, o fato de não poderem reconhecer visualmente o agressor dificultam a denúncia e facilitam a impunidade.

A Política Nacional de Saúde para as pessoas com necessidades especiais adota o conceito fixado pelo Decreto n. 3.298/99, que considera pessoa com deficiência aquela que apresenta, em caráter permanente, perdas ou anormalidades de sua estrutura ou função psicológica, fisiológica ou anatômica, que geram incapacidade para o desempenho de atividades dentro do padrão considerado normal para o ser humano ${ }^{(7)}$.

O fato de apresentar uma deficiência agrava a vulnerabilidade feminina, visto que essas encontram-se mais suscetíveis à violência sexual ${ }^{(8)}$. Diante desse quadro é importante ressaltar que na Constituição Brasileira estão assegurados os direitos das pessoas com deficiências nos mais diferentes campos e aspectos ${ }^{(9)}$.

Face ao exposto, esse estudo é relevante porque, além do reduzido número de pesquisas sobre esta temática, vem se somar à luta de alguns autores ${ }^{(10-11)}$ que defendem a integridade sexual da pessoa com necessidade especial, independente das limitações física, sensorial ou intelectual que possam acarretar. Portanto, o objetivo desta pesquisa foi caracterizar o perfil da vítima e do agressor da violência sexual a portadoras de necessidades especiais.

\section{MÉTODO}

Estudo de abordagem quantitativa, transversal e descritivo, utilizou dados secundários e foi desenvolvido no Núcleo de Medicina e Odontologia Legal (NUMOL) de Campina Grande, Paraíba, instituição para onde são encaminhadas as vítimas de violência sexual, com o objetivo de realizar o exame de conjunção carnal para a quantificação e a qualificação de lesões. O exame de conjunção carnal consiste em um conjunto de vestígios deixados pelo fato delituoso, são os elementos materiais perceptíveis pelos sentidos e embasam as perícias. O resultado do exame é registrado em um laudo médico ${ }^{(12)}$.

O universo pesquisado englobou 1.101 laudos médicos e respectivos Boletins de Ocorrência de exames de conjunção carnal registrados no período de 2004 a 2009, totalizando 2.202 documentos analisados. Foram incluídos no estudo apenas os casos confirmados de estupro em mulheres com necessidades especiais $(\mathrm{n}=396)$.

$\mathrm{O}$ instrumento de pesquisa consistiu de um formulário específico, desenvolvido a partir do laudo médico e dos boletins de ocorrência, contendo questões abertas e fechadas, dicotômicas e de múltipla escolha. As variáveis de estudo foram: 1) relativas à vítima: idade, faixa etária, estado civil, escolaridade e tipo de necessidade especial; 2) relativas à denúncia: ano, denunciante e encaminha- 
mento ao NUMOL; 3) relacionados ao agressor: relação com a vítima, grau de relacionamento e número de agressores; 4) relacionadas à agressão: local, data provável da conjunção carnal, existência de outro tipo de violência durante a prática e forma de constrangimento utilizada.

Previamente à coleta de dados os pesquisadores testaram o instrumento de pesquisa por meio de um estudo piloto, objetivando verificar a existência de erros ou falhas. Os dados foram coletados por dois pesquisadores no período de março a agosto de 2010.

O banco de dados e as análises estatísticas foram realizados no software SPSS 17.0 (Statistical Package for the Social Sciences). Os dados foram apresentados por meio da estatística descritiva (distribuições absolutas e percentuais).

Conforme os preceitos éticos vigentes estabelecidos na Resolução 196/96, o estudo foi registrado no Sistema Nacional de Informação sobre Ética em Pesquisa envolvendo Seres Humanos - SISNEP e aprovado pelo Comitê de Ética em Pesquisa da Universidade Estadual da Paraíba, sob número 0538.0.133.000-09.

\section{RESULTADOS}

Do total de 1.101 laudos analisados, 396(36,0\%) tiveram a conjunção carnal confirmada. No entanto, apenas $19(4,8 \%)$ laudos faziam referência a tratar-se de mulheres portadoras de necessidades especiais. A idade das vítimas variou de 12 a 28 anos, média de 16,6 anos $( \pm 5,33)$, com mais de dois terços das mulheres na segunda década de vida $(68,4 \%)$. Com relação à escolaridade, 26,3\% eram alfabetizadas (Tabela 1). Todas as vítimas eram solteiras, a maioria residente na zona rural $(52,6 \%)$ e natural de outros municípios $(63,2 \%)$.

No tocante ao tipo de necessidade especial, em apenas seis laudos havia o registro dessa informação, a maioria com deficiência intelectual ( $n=5 ; 83,33 \%)$, o outro caso registrou que a vítima portava múltiplas necessidades especiais: surdez, mudez, física e intelectual. Os demais laudos, a despeito de possuírem o registro de que a vítima era portadora de algum tipo de necessidade especial, não apresentavam qual necessidade.

Em qualquer situação de violência, o denunciante é um personagem extremamente importante. No presente trabalho, foi possível identificar o autor da denúncia em apenas três laudos $(15,8 \%)$, sendo em todos eles a genitora da vítima. Todas as vítimas foram encaminhadas ao NUMOL pelas delegacias nas quais o registro da ocorrência se efetuou.

A análise do agressor revelou que a maioria $(78,9 \%)$ era conhecida da vítima. Quanto ao número de agressores predominou um único perpetrador em 73,7\% dos casos, enquanto que para $15,8 \%$ das vítimas os autores da violência foram dois ou mais indivíduos. Em dois laudos (10,5\%) não constava o registro dessa informação (Tabela 2).

O local onde a violência sexual ocorreu não foi identificado em $78,9 \%$ dos laudos, como mostrado na Tabela 3. Em relação ao tempo de ocorrência da violência, $63,2 \%$ tinham ocorrido há mais de 20 dias e a maioria das vítimas eram virgens antes da agressão (52,6\%). Ao se analisar a existência de constrangimento durante a prática do estupro, observou-se que em $21,1 \%$ dos casos ocorreu, sendo a força física e a grave ameaça as duas mais frequentes (Tabela 2).

Tabela 1 - Distribuição de mulheres portadoras de necessidades especiais vítimas de violência sexual segundo a faixa etária e escolaridade. Campina Grande, 2004 - 2009

\begin{tabular}{llcc}
\hline \multicolumn{1}{c}{ VARIÁVEIS } & & \multicolumn{2}{c}{ FREQUENCIA } \\
\hline \multirow{3}{*}{ Faixa etária } & & n & \% \\
& $12-19$ anos & 13 & 68,4 \\
& $20-28$ anos & 5 & 26,3 \\
& NR* & 1 & 5,3 \\
& Total & 19 & 100 \\
& & & \\
& Analfabeta & 1 & 5,3 \\
& Alfabetizada & 5 & 26,3 \\
& Ensino fundamental incompleto & 1 & 5,3 \\
& Ensino fundamental completo & 3 & 15,8 \\
& Ensino médio incompleto & 2 & 10,5 \\
& NR* & 7 & 36,8 \\
& Total & 19 & 100,0 \\
\hline
\end{tabular}

*NR - laudo sem a informação 
Tabela 2 - Distribuição de mulheres portadoras de necessidades especiais vítimas de violência sexual segundo o grau de parentesco com o agressor, o número de agressores e características da ocorrência. Campina Grande, 2004 - 2009

\begin{tabular}{|c|c|c|c|}
\hline \multirow[t]{2}{*}{ VARIÁVEIS } & & \multicolumn{2}{|c|}{ FREQUENCIA } \\
\hline & & n & $\%$ \\
\hline \multirow{5}{*}{$\begin{array}{l}\text { Grau de parentesco/ } \\
\text { Relação com a vítima }\end{array}$} & Pai biológico & 1 & 5,3 \\
\hline & Atual parceiro & 2 & 10,5 \\
\hline & Outros conhecidos* & 12 & 63,2 \\
\hline & $\mathrm{NA}^{* *}$ & 4 & 21,1 \\
\hline & Total & 19 & 100,0 \\
\hline \multirow{4}{*}{ Número de agressores } & Único & 14 & 73,7 \\
\hline & Múltiplo & 3 & 15,8 \\
\hline & $\mathrm{NR}^{* * *}$ & 2 & 10,5 \\
\hline & Total & 19 & 100,0 \\
\hline \multirow{3}{*}{ Local do estupro } & Residência do agressor & 2 & 10,5 \\
\hline & Local público & 2 & 10,5 \\
\hline & $\mathrm{NR}^{* * *}$ & 15 & 78,9 \\
\hline \multirow{3}{*}{ Tempo da ocorrência } & $<20$ dias & 6 & 31,6 \\
\hline & $>20$ dias & 12 & 63,2 \\
\hline & $\mathrm{NR}^{* * *}$ & 1 & 5,3 \\
\hline \multirow{3}{*}{ Vítima virgem } & Sim & 10 & 52,6 \\
\hline & Não & 5 & 26,3 \\
\hline & $\mathrm{NR}^{* * *}$ & 4 & 21,1 \\
\hline \multirow{3}{*}{ Constrangimento } & Sim & 4 & 21,1 \\
\hline & Não & 1 & 5,3 \\
\hline & $\mathrm{NR}^{* * *}$ & 14 & 73,7 \\
\hline \multirow{3}{*}{$\begin{array}{l}\text { Forma de } \\
\text { constrangimento }\end{array}$} & Força física & 2 & 10,5 \\
\hline & Força física e grave ameaça & 2 & 10,5 \\
\hline & $\mathrm{NR}^{* * *}$ & 15 & 78,9 \\
\hline
\end{tabular}

NR: laudo com informação ausente; *Outros conhecidos: pessoa que a vítima conversa, cumprimenta ou sabe o nome. Não possuí grau de parentesco; **NA: não se aplica; ***NR: laudo sem a informação.

\section{DISCUSSÃO}

O presente estudo reforça a compreensão da violência sexual como problema de saúde pública de grande magnitude. Demanda uma abordagem que contemple a integralidade e a interdisciplinaridade para lidar com os significativos impactos físicos, subjetivos, sexuais e afetivos na vida das mulheres com necessidades especiais.

Apesar do número restrito de casos do estudo, as informações obtidas ressaltam a importância da prevenção da violência sexual entre mulheres portadoras de necessidades especiais. Dessa maneira, essas pessoas poderiam receber a ajuda necessária para que, diante de situações potencialmente abusivas, fossem capazes de se proteger e não sofrer violência sexual. Sendo assim, essa população terá seus direitos garantidos e suas chances de terem uma vida mais saudável e plena.

Dos dezenoves laudos que tratam de pessoas com deficiência, apenas três traziam informações acerca de quem foi o denunciante da violência. Os casos de violência que chegam ao estágio da "denúncia" refletem ainda a minoria, tornando-os inconsistentes diante da grandiosidade real do problema e expõem uma falsa situação. Há, entretanto, que se ter cautela em relação à frequência do abuso em pessoas 
com necessidades especiais visto que além de haver carência de dados nesta área de pesquisa, a prevalência varia consideravelmente dependendo do estudo ${ }^{(13)}$.

A Lei n. 11.340/2006 traz em seu capítulo III que no atendimento à mulher em situação de violência, a autoridade policial deverá, entre outras providências: garantir proteção policial, quando necessário, comunicando de imediato ao Ministério Público e ao Poder Judiciário; encaminhar a ofendida ao hospital ou posto de saúde e ao Instituto Médico Legal, fornecer transporte para a ofendida e seus dependentes para abrigo ou local seguro, quando houver risco de vida ${ }^{(3)}$.Contudo, ao se analisar quem são os agressores, na maioria pessoas com parentesco e conhecidos, possivelmente moradores na mesma residência, há de se refletir as dificuldades para a garantia da segurança das vítimas.

Nesse estudo, a faixa etária com maior frequência de casos foi dos 12 aos 19 anos, prevalecendo, portanto, a fase do desenvolvimento humano que marca a transição entre a adolescência e a idade adulta, característica esta já observada previamente ${ }^{(14)}$. O fato das vítimas serem solteiras reforça a ideia que elas são indefesas, dependentes e carentes de cuidados. Tal vulnerabilidade se justifica a partir de relações que poderiam ser de confiança e proteção, mas que, no entanto, são usadas como subterfúgio para a violência ${ }^{(12)}$. Estudo prévio demonstrou que as mulheres que se declararam civilmente solteiras, com companheiros, foram as maiores vítimas da violência física e também estiveram mais expostas à violência psicológica, bem como à violência estrutural ${ }^{(15)}$.

O baixo grau de escolaridade e o fato do maior número de vítimas residirem na zona rural demonstram que estas possuem um menor grau de conhecimento. Nas classes sociais menos favorecidas, a violência pode ser fruto do baixo nível educacional, de uma lamentável tradição cultural, do desemprego, do uso de drogas e alcoolismo. O conceito de fragilidade da mulher é oriundo de todo um desenvolvimento histórico, que a colocou em posição submissa frente ao homem, vulnerável a ele e seus comportamentos hostis ${ }^{(15)}$.

Dentre as vítimas, predominou as portadoras de necessidade intelectual. Esse grupo de portadoras é vítima de abuso em maior proporção do que as pessoas da população em geral ${ }^{(16)}$. Em se tratando especificamente do abuso sexual, a mulher com deficiência intelectual sofre maior risco do que a mulher não portadora em função de dificuldades no julgamento e em escassas habilidades sociais que resultam em maior vulnerabilidade; dificuldades de comunicação; inabilidade em buscar ajuda ou em denunciar o abuso; falta de conhecimento em como se defender do abuso; falta de conhecimento sobre comportamento sexual apropriado; dependência excessiva de terceiros ${ }^{(17)}$.

Verificou-se que em muitos casos os agressores eram conhecidos da vítima, prevalecendo aqueles sem parentesco familiar, sendo na maioria das vezes um único agressor. Outros autores afirmaram serem os maridos ou companheiros os principais perpetradores ${ }^{(18)}$, de modo que muitas mulheres reconhecem e relatam sofrimento psicológico permeado durante a relação ${ }^{(19)}$.

O local de realização da agressão não havia sido registrado na maioria dos laudos, impossibilitando a análise. A violência contra a mulher se manifesta de diversas formas e ocorre tanto no âmbito familiar, como em locais públicos ou privados, no trabalho ou na própria comunidade ${ }^{(18)}$.

Faz-se necessário que se entenda que, mesmo não havendo sequelas físicas, as consequências da violência psicológica são profundas, com marcas que vão muito além das lesões aparentes ${ }^{(19-20)}$.

O presente estudo apresentou algumas limitações, como a ausência de registro de algumas informações importantes nos laudos e o pequeno número de casos confirmados da violência sexual em mulheres com necessidades especiais. Este fato denota falha no preenchimento desse documento e indica uma possível subnotificação da violência sexual neste grupo de pessoas.

Em face dos resultados é importante a formação de Conselhos locais para detecção e supressão da violência, incluindo o desenvolvimento de atividades de educação em saúde em distintos locais (escolas, unidades de saúde, clubes de mães, de trabalhadores, dentre outros), objetivando a orientação acerca do problema e como detectar esse tipo de violência por meio da observação de alterações comportamentais sugestivas de sofrimento psicológico pelo abuso sexual.

\section{CONSIDERAÇÕES FINAIS}

O estudo demonstrou que a violência sexual envolvendo portadoras de necessidades especiais no município de Campina Grande acometeu mulheres jovens, de baixa escolaridade, possuidoras de deficiência intelectual, e que os agressores pertencem ao círculo social da vítima e agem isoladamente na maioria das situações.

Apesar dos avanços alcançados na assistência a mulheres vítimas de violência sexual o atendimento integral ainda é considerado um desafio para os órgãos públicos, em especial quando se trata de pacientes portadoras de necessidades especiais. Estas apresentam limitações que 
as tornam mais expostas à violência e a impunidade.

Apesar do número de vítimas ser relativamente baixo, se sugere atenção especial, visto que muitos fatores podem interferir negativamente no número de denúncias, determinando sub-notificação, desde a decisão denunciar.

Conclui-se pela necessidade de profissionais, principalmente os que atuam com atendimento ao público, como é o caso dos enfermeiros e técnicos de enfermagem que trabalham em hospitais e unidades de saúde, estejam atentos e preparados para atender e suprir as necessidades desta demanda, considerando que a realidade da violência no cotidiano dessas mulheres é fato evidente e relevante na literatura, porém, ainda assim, continua sendo invisível.

\section{REFERÊNCIAS}

1. Villela WV, Lago T. Conquistas e desafios no atendimento das mulheres que sofreram violência sexual. Cad. saude publica. 2007;23(2):471-5.

2. Ministério da Saúde (BR). Secretaria Especial de Políticas para as Mulheres. Política Nacional de Enfrentamento à Violência contra as Mulheres. Brasília. 2003.

3. Ministério da Saúde (BR). Seguridade Social e Direitos Humanos. Centro de Direitos Humanos. Coleção Cartilhas Sobre Direitos Humanos. Gênero e Direitos Humanos. Brasília: Ministério da Saúde; 2005.

4. Ministério da Saúde (BR). Secretaria de Atenção à Saúde. Departamento de Ações Programáticas Estratégicas. Diretos sexuais, reprodutivos e métodos anticoncepcionais. Brasília: Ministério da Saúde; 2009.

5. Cursino HM, Rodrigues OMPR, Maia ACB, Palamin MEG. Orientação sexual para jovens adultos com deficiência auditiva. Rev. bras. educ. espec. 2006;12(1):29-48.

6. Pagliuca LMF, Regis CG, França ISX. Análise da comunicação entre cego e estudante de Enfermagem. Rev. bras. enferm. 2008; 61(3):296-301.

7. Ministério da Saúde (BR). Secretaria de Atenção à Saúde. Departamento de Ações Programáticas e Estratégicas. Área Técnica Saúde da Pessoa com Deficiência. Política nacional da pessoa portadora de deficiência. Brasília: Ministério da Saúde; 2008.

8. Ministério da Saúde (BR). Secretaria de Atenção à Saúde. Departamento de Ações Programáticas Estratégicas. Diretos sexuais, reprodutivos e métodos anticoncepcionais. Brasília: Ministério da Saúde; 2009.

9. Brasil. Constituição da República Federativa do Brasil. Brasília: Senado; 1988.

10. Cruz DMC, Silva JT, Alves HC. Evidências sobre violência e deficiência: implicações para futuras pesquisas. Rev. bras. educ. espec. 2007;13(1):131-46.

11. Ribeiro WS, Andreoli SB, Ferri CP, Prince M, Mari JJ. Exposição à violência e problemas de saúde mental em países em desenvolvimento: uma revisão da literatura. Rev. bras. psiquiatr. 2009;31 Suppl 2:S49-57.

12. França GV. Fundamentos de medicina legal. Rio de Janeiro: Guanabara Koogan; 2005.

13. Baptista R, Vieira N, Ximenes L, Pagliuca L. Analysis the legislation for disabled people in health promotion. OBJN [Internet] 2010; 9(1) [acesso em 15 de jul 2011]. Disponível: http://www.objnursing.uff.br/index.php/ nursing/article/view/j.1676-4285.2010.2762

14. Diniz NMF, Almeida LCG, Ribeiro BCS, Macedo VG. Women victims of sexual violence: adherence to chemoprevention of HIV. Rev. latino-am. enfermagem. 2007;15(1):7-12.

15. Lopes IMRS, Gomes KRO, Silva BB, Deus MCBR, Galvão ERCG, Borba DC. Caracterização da violência sexual em mulheres atendidas no projeto Maria-Maria em Teresina-PI. Rev. bras. ginecol. obstet. 2004; 26(2):111-6.

16. Strickler HL. Interaction between family violence and mental retardation. Ment. retard. 2001;39(6):461-71.

17. Barros RD, Williams LCA, Brino RF. Habilidades de auto proteção acerca do abuso sexual em mulheres com deficiência mental. Rev. bras. educ. espec. 2008;14(1):93-110.

18. Mattar R, Abrahão AR, Andalaft Neto J, Colas OR, Schroeder I, Machado SJR, et al. Assistência multiprofissional à vítima de violência sexual: a experiência da Universidade Federal de São Paulo. Cad. saúde pública. 2007;23(2):459-64.

19. ParenteEO, Nascimento RO, Vieira LJES. Enfrentamento da violência doméstica por um grupo de mulheres após a denúncia. Rev. estud. fem. 2009;17(2):445-65.

20. Ferraz MIR, Labronici LM. Perfil da violência doméstica contra a mulher em Guarapuava, Paraná. Cava, Paraná. Cogitare enferm. 2009;14(2):261-8. 